\title{
Comunicação de Notícias Difíceis: Revisão Integrativa Sobre Estratégias de Ensino na Formação Médica
}

\section{Communication of Difficult News: Integrative Review on Teaching Strategies in Medical Education}

\section{Comunicación de Noticias Difíciles: Revisión Integradora Sobre Estrategias de Enseñanza en Educación Médica}

\author{
Esther Almeida da Silva-Xavier ${ }^{1}$ \\ Larissa Polejack \\ Eliane Maria Fleury Seidl \\ Universidade de Brasilia (UnB)
}

\begin{abstract}
Resumo
Notícias difíceis são informações que afetam seriamente a visão do indivíduo sobre o seu futuro. Na saúde, são atribuições médicas em situações de comunicação diagnóstica de condições clínicas graves, no prognóstico e/ou terapêutica desfavorável de quadros irreversíveis ou sem tratamento modificador (falecimentos, sequelas, assistência paliativa). Este estudo realizou revisão integrativa das estratégias de ensino desse tipo de comunicação na formação médica. Foram acessadas a PsycINFO, a Biblioteca Virtual em Saúde e a PubMed, de 2014 até maio de 2019. Foram encontrados 59 resumos e, após aplicação dos critérios de exclusão, 13 artigos empíricos foram analisados. Identificou-se diversidade no uso de estratégias de ensino, sendo a simulação (com atores ou pessoas treinadas) a mais descrita. Outras estratégias (discussão entre pares, escrita reflexiva, reunião compartilhada com pacientes, vídeos de casos e emprego de tecnologias digitais) também foram identificadas. O uso combinado dessas estratégias tem importância primordial no treinamento profissional contemporâneo.

Palavras-chave: educação médica, notícias difíceis, ensino-aprendizagem
\end{abstract}

\begin{abstract}
Difficult news are informations that seriously affect an individual's view of their future. In health, they are medical assignments in situations of diagnostic communication of serious clinical conditions, in the prognosis and/or unfavorable treatment of irreversible conditions or without modifying treatment (deaths, sequelae, palliative care). This study carried out an integrative review of teaching strategies for this type of communication in medical education. PsycINFO, the Virtual Health Library, and PubMed were accessed from 2014 to May 2019. We found fifty-nine abstracts, and after applying the exclusion criteria, we analyzed 13 empirical articles. Diversity in the use of teaching strategies was identified, with simulation (with actors or trained people) being the most described. Other strategies (peer discussion, reflective writing, shared meeting with patients, case videos, and use of digital technologies) were also reported. The combined use of these teaching-learning strategies is of paramount importance in contemporary professional training.

Keywords: medical education, difficult news, teaching-learning
\end{abstract}

\section{Resumen}

Las noticias difíciles son informaciones que afectan seriamente la visión de un individuo sobre su futuro. En salud, son asignaciones médicas en situaciones de comunicación diagnóstica de afecciones clínicas graves, en el pronóstico y/o tratamiento desfavorable de afecciones irreversibles o sin modificar el tratamiento (muertes, secuelas, cuidados paliativos). Este estudio llevó a cabo una revisión integradora sobre estrategias de enseñanza para ese tipo de comunicación en educación médica. Se accedió el PsycINFO, la Biblioteca Virtual de Salud y el PubMed, de 2014 a mayo de 2019. Se encontraron 59 resúmenes y, después de aplicar los criterios de exclusión, se analizaron 13 artículos empíricos. Se identificó la diversidad en el uso de estrategias de enseñanza, siendo la simulación (con actores o personas capacitadas) la más descrita. También se informaron otras estrategias (discusión entre pares, escritura reflexiva, reunión compartida con pacientes, videos de casos y uso de tecnologías digitales). El uso combinado de estas estrategias de enseñanza-aprendizaje es de suma importancia en la formación profesional contemporánea.

Palabras clave: educación médica, noticias difíciles, enseñanza-aprendizaje

\footnotetext{
${ }^{1}$ Endereço de contato: Quadra 204, lote 2, Ed. Alfamix Center, sala 414, Águas Claras, Brasília-DF, CEP: 71939-540. E-mail: esther.gea.ex@gmail.com
} 


\section{Introdução}

A comunicação é uma atividade básica da experiência humana e uma importante ferramenta relacional em nossas vidas. Sua origem etimológica é latina (communicatio), sendo traduzida literalmente como "tornar comum" e sendo-Ihe atribuído o significado do ato de repartir, dividir, distribuir (Cunha, 2010). Nenhum grupo social existe sem ela, de modo que cabe à comunicação tanto a troca quanto o compartilhamento de ideias e informações entre seus membros. Quando inadequada, torna-se fonte de conflitos interpessoais. Para que se torne eficiente, é importante que contemple aspectos motivacionais e proporcione expressão emocional e afetiva, gerando informação para a tomada de decisão que auxiliará na regulação do comportamento do grupo (Robbins, 2004).

Como a saúde e a doença são experiências íntimas em nossa vivência humana, ambas demandam uma contextualização sociocultural por meio da comunicação. Nas trocas comunicativas, a saúde e o adoecimento dos sujeitos interatuam de modo particular, estabelecendo uma relação clínica. Esta, quando estabelecida a partir de uma comunicação adequada, torna-se fundamental para o compartilhamento de informações diagnósticas, terapêuticas e bioéticas, fortalecendo também os laços de empatia e de confiança dos envolvidos nessa relação (Ruiz, 2003). No atual cenário da medicina, a comunicação é um aspecto essencial do relacionamento clínico, isto porque ela possibilita a construção intersubjetiva inerente à atuação médica e à relação com o paciente (Stellyes, 2017). Assim, nessa área, a comunicação se torna eixo condutor, tanto para as notícias boas quanto para as indesejadas.

A comunicação de más notícias é uma tarefa importante na rotina dos profissionais de saúde, apesar de não ser a preferida pela maioria. Essa modalidade de comunicação versa sobre conteúdos e/ou temas que constituem situações de ameaça à vida, ao bem-estar pessoal, familiar e social, devido a repercussões físicas, sociais e emocionais que causam ao paciente e à família. Uma má notícia pode ser definida como qualquer informação que afete seriamente e de forma adversa a visão de um indivíduo sobre o seu futuro. Na saúde, ela relaciona-se a condições clínicas graves, crônicas, irreversíveis ou sem possibilidade de terapia curativa ou tratamento modificador (Araújo \& Leitão, 2012; Baile et al., 2000; Chehuen Neto et al., 2013).

A comunicação de más notícias deve sempre ser contextualizada na perspectiva de quem a experiencia, de modo que não há como calcular de antemão seu dano até que se tenha determinado as expectativas e a compreensão de quem a recebe (Baile et al., 2000). Desse modo, este trabalho optou por adotar o termo notícia difícil, em vez de má notícia, já que este último pode exacerbar um juízo de valor prévio, adicionando um cunho pejorativo à informação compartilhada.

Comunicar notícias difíceis é tarefa árdua, podendo desencadear conflitos morais e questionamentos éticos relacionados à autonomia e ao livre-arbítrio na relação estabelecida entre o profissional, a família e o paciente (Geovanini \& Braz, 2013). Como esse tipo de comunicação pode acontecer em cenários complexos permeados por insegurança, medo e desconhecimento, o adequado manejo do assunto pelos profissionais envolvidos torna-se uma necessidade. Após recebimento de uma notícia difícil, a maior parte das pessoas pode expressar sentimentos negativos, o que pode ser agravado quando a comunicação é feita de maneira inapropriada. Para se evitar isso, a capacitação profissional médica é fundamental. Ademais, esse preparo é necessário, pois a comunicação diagnóstica é uma das atribuições descritas no Código de Ética Médica, capítulo V, artigo 34, que veda ao profissional "deixar 
de informar ao paciente o diagnóstico, o prognóstico, os riscos e os objetivos do tratamento, salvo quando a comunicação direta possa Ihe provocar dano, devendo, nesse caso, fazer a comunicação a seu representante legal" (Brasil, 2009, p. 38).

A comunicação terapêutica em saúde nem sempre é trabalhada na graduação e na pós-graduação médica, sendo aprendida na prática e, muitas vezes, sem reflexões sistemáticas e regulares. Nesse caso, não é incomum que as habilidades de comunicação sejam ensinadas por meio da abordagem "veja um, faça um, ensine um" - "see one, do one, teach one" approach (Lamba, Tyrie, Bryczkowski, \& Nagurka, 2016, p. 105). Essa abordagem não costuma priorizar as implicações emocionais do processo, pautando-se na execução operacional dos procedimentos e condutas. Para tanto, é necessário trabalhar também as emoções e os sentimentos envolvidos no processo de comunicação de notícias difíceis; pois, quando o profissional identifica a emoção experienciada, ele consegue adequar sua atuação e melhorar seu desempenho junto a pacientes e familiares, promovendo, assim, uma permanência saudável em sua área de atuação (Afonso \& Minayo, 2017).

Um estudo qualitativo finlandês identificou que a comunicação de notícias difíceis é um evento estressante, não só para os médicos em exercício com longa experiência, mas também para os estudantes. Os resultados sinalizaram uma clara tensão entre sentir empatia e ter distância profissional. O distanciamento emocional foi descrito como contraditório aos pensamentos empáticos dos estudantes. Devido a isso, a supressão emocional e a redução propositiva da empatia foram empregadas como mecanismos de enfrentamento, sendo o distanciamento também usado como uma estratégia para gerenciar o estresse da comunicação de notícias difíceis. Esses achados sugerem falta de estratégias de enfrentamento adequadas para manejo de encontros desafiadores, e o estudo discute que sentimentos desconcertantes devem ser abordados e os alunos devem receber apoio com relação a esses desafios. Assim, faz-se necessário o emprego de meios educacionais para desenvolver a empatia e o enfrentamento por meio de aprendizagem experiencial, humanidades médicas, além de intervenções baseadas em escrita reflexiva e em grupo (Toivonena, LindblomYlänneb, Louhialaa, \& Pyöräläc, 2017).

Segundo um estudo brasileiro sobre a percepção médica quanto à comunicação de notícias difíceis em Oncologia, não há uma capacitação apta a dirimir por completo os sentimentos negativos envolvidos nesse processo. Isto porque é evidente a existência de algum grau de sofrimento no paciente que recebe uma notícia difícil, assim como no profissional que a comunica, especialmente em situações de finitude de vida ou de cessão terapêutica curativa. Todavia, a abordagem desse tema e o reconhecimento de sua complexidade são oportunidades para aprender a lidar com os sofrimentos inerentes à morte e ao morrer. Para tanto, ressalta-se a necessidade de se implantarem estratégias educacionais que vão ao encontro de uma formação médica sincronizada com o processo de humanização assistencial em saúde (Silva, Sousa, \& Ribeiro, 2018).

Conforme os pontos elencados no presente artigo, depreende-se que, se a comunicação terapêutica padece de preparo, tanto na graduação quanto nas residências médicas, não é de se estranhar que isso também ocorra no cenário de comunicação de notícias difíceis. Essa situação não é circunscrita apenas à realidade brasileira, pois um estudo norte-americano de revisão integrativa identificou lacunas na literatura quanto ao ensino e ao preparo 
dos residentes cirúrgicos para esse tipo de comunicação em ambiente de trauma e de centro cirúrgico, após eventos inesperados. Além da dificuldade em manejar essa situação, os resultados da revisão apontaram dificuldades dos cirurgiões em repassar notícias aos familiares, especialmente quando não havia uma relação médico-paciente previamente estabelecida no contexto do evento traumático (Lamba et al., 2016).

Diante do exposto e com as mudanças na sociedade que envolvem o uso de novas tecnologias e o acesso à informação, o campo biomédico sofre o impacto desse processo demandando a reformulação dos paradigmas de cuidado em saúde. Não desconsiderando os avanços farmacológicos, o grande rol de mudanças na área biomédica centra-se na alteração do paradigma estabelecido na relação médico-paciente, especialmente no que tange ao lugar da revelação de informações fidedignas nesse ínterim. Cada vez mais, os pacientes ocupam um espaço diferenciado e menos passivo, enquanto os profissionais de saúde seguem sendo formados para não lidar com essa realidade (Geovanini \& Braz, 2013).

O preparo médico para comunicar notícias difíceis desponta com premência no cenário atual, em que pacientes buscam informações diagnósticas na internet. Isso inclusive repercute na relação clínica, demandando novas formas de se relacionar e de se comunicar em saúde. Diante disso, uma proposta viável para se lidar com essa situação seria a adoção de metodologias ativas de ensino na formação dos profissionais. A partir disso, Paiva, Parente, Brandão e Queiroz (2016) sinalizam que:

Os procedimentos de ensino são tão importantes quanto os próprios conteúdos de aprendizagem. Portanto, as técnicas de ensino tradicional passam a fazer parte do escopo de teóricos não só da área da Educação, mas de toda a comunidade intelectual que busca identificar suas deficiências e buscam [sic] propor novas metodologias de ensino-aprendizagem. (p. 146).

Uma característica comum entre as metodologias ativas de ensino é a concepção de educação problematizadora, em que o aprendiz é instigado a se implicar com o mundo a sua volta, elaborando uma compreensão acerca de sua realidade. Isso, por sua vez, possibilita-Ihe intervir nessa realidade, transformando-a. Essa perspectiva rompe com o modelo tradicional centrado na abordagem conteudista, com enfoque individual no processo de ensino (Paiva et al., 2016). Nas metodologias ativas, o aluno é o centro do processo de aprendizagem, contrapondo-se à ideia de espectador. Nela, o ensino nasce na prática e busca uma teoria que o explique. Com isso, há uma mudança do "ensinar" para o "aprender", havendo um desvio do foco do docente para o aluno, que se corresponsabiliza por seu aprendizado (Souza, Iglesias, \& Pazin-Filho, 2014).

A metodologia ativa trabalha com situações-problema, de modo que o docente necessita ter conhecimento da temática e do cenário e ter experiência com essas situações, a fim de instigar a curiosidade dos discentes. Essas metodologias problematizam a realidade, possibilitando a aprendizagem a partir da cena e do contexto em que o aprendiz se encontra inserido. Isso faz com que ele interaja com o seu processo de aprendizado, exercitando habilidades futuras de diálogo, observação, reflexão crítica e de tomada de decisão quanto à conduta a ser adotada (Diesel, Baldez, \& Martins, 2017). Em uma revisão integrativa brasileira, identificou-se predomínio do uso de metodologias ativas de ensino-aprendizagem no ensino superior, principalmente no contexto de educação em saúde (Paiva et al., 2016). Disso, infere-se que as metodologias ativas contribuem na formação médica, ao promover o contato 
do estudante ou do profissional com vivências cotidianas ou limítrofes de sua profissão, em um ambiente preparado e protegido de ensino.

Diante do exposto, o presente estudo teve por objetivo identificar qual metodologia ativa ou estratégia de ensino foi descrita na formação médica para comunicação de notícias difíceis, no período de 2014 a maio de 2019, voltada tanto para estudantes de graduação quanto para residentes ou médicos.

\section{Método}

Trata-se de uma revisão integrativa (RI) da literatura que visou sintetizar e analisar a produção científica de artigos empíricos sobre o uso de metodologias ativas na formação médica para comunicação de notícias difíceis. Optou-se pela RI, pois esta viabiliza a organização do conhecimento científico, possibilitando ao pesquisador contatar outros trabalhos e questões similares àquelas que ele se propõe a investigar, assim delineando o cenário científico do tema a ser estudado. Ademais, isso possibilita pensar outras oportunidades a serem trabalhadas, já que oferta informações sobre procedimentos usados em trabalhos prévios (Botelho, Cunha, \& Macedo, 2011). Por se caracterizar como uma sinopse dos principais pontos de um conceito ou área de estudo, a RI reúne e sintetiza métodos, teorias e/ou estudos empíricos, tendo abrangência ampla ou limitada em pesquisas quantitativas, qualitativas ou de métodos mistos. Seu uso é versátil na definição de conceitos, na revisão de teorias e evidências, bem como na análise de problemas metodológicos de um tema específico (Ercole, Melo, \& Alcoforado, 2014; Souza, Silva, \& Carvalho, 2010).

Inicialmente, foi realizada a definição do problema a partir de revisão bibliográfica, buscando identificar artigos empíricos nas seguintes bases de dados: PsycINFO, base de dados da American Psychological Association (APA) e nos portais da Biblioteca Virtual em Saúde (BVS) e da PubMed, a partir da fórmula de descritores: ("medical education") AND ("bad news" OR "difficult news") AND ("learning"). Os descritores foram definidos conforme o Medical Subject Headings (MeSH) e os Descritores em Ciências da Saúde (DeCS).

Foram considerados elegíveis os artigos disponíveis na íntegra, publicados em português ou inglês, no período de 2014 até o 1오 semestre 2019, precisamente maio. Foram excluídas outras publicações científicas (editoriais, comentários, artigos de revisão, relatos de experiência, teses, dissertações, artigos de profissionais não médicos), manuscritos que não apresentassem informações quanto ao uso de metodologias ativas no resumo ou trabalhos que tivessem por objetivo a validação de instrumentos para avaliar a comunicação de notícias difíceis.

As informações coletadas dos artigos foram agrupadas em uma planilha Excel, constando link e DOI, a referência conforme a APA, palavras-chave, país do estudo, base de dados ou portal identificados, título, idioma, ano de publicação, delineamento do estudo, objetivo(s), participantes, método, resultados principais, discussão/comentários. Por fim, foi realizada a análise dos resultados com a síntese dos artigos selecionados de maneira descritiva, possibilitando apresentação da metodologia ativa levantada ou das estratégias de ensino, conforme o objetivo deste estudo. 


\section{Resultados}

Foram identificados 59 resumos nas bases de dados e nos portais selecionados. Após aplicação dos critérios de exclusão, foram analisados 13 artigos empíricos, sendo 12 em língua inglesa e um em língua portuguesa (Figura 1). Quanto ao ano de publicação: três são de 2014; dois de 2015; um de 2016; quatro de 2017 e três de 2018. No que se refere ao país de realização dos estudos, seis eram dos Estados Unidos, dois eram estudos transculturais realizados entre o Canadá, a China e os Estados Unidos, um era de Israel, um da Finlândia, dois da Suíça e um do Brasil.

Figura 1. Flow diagram da seleção dos artigos

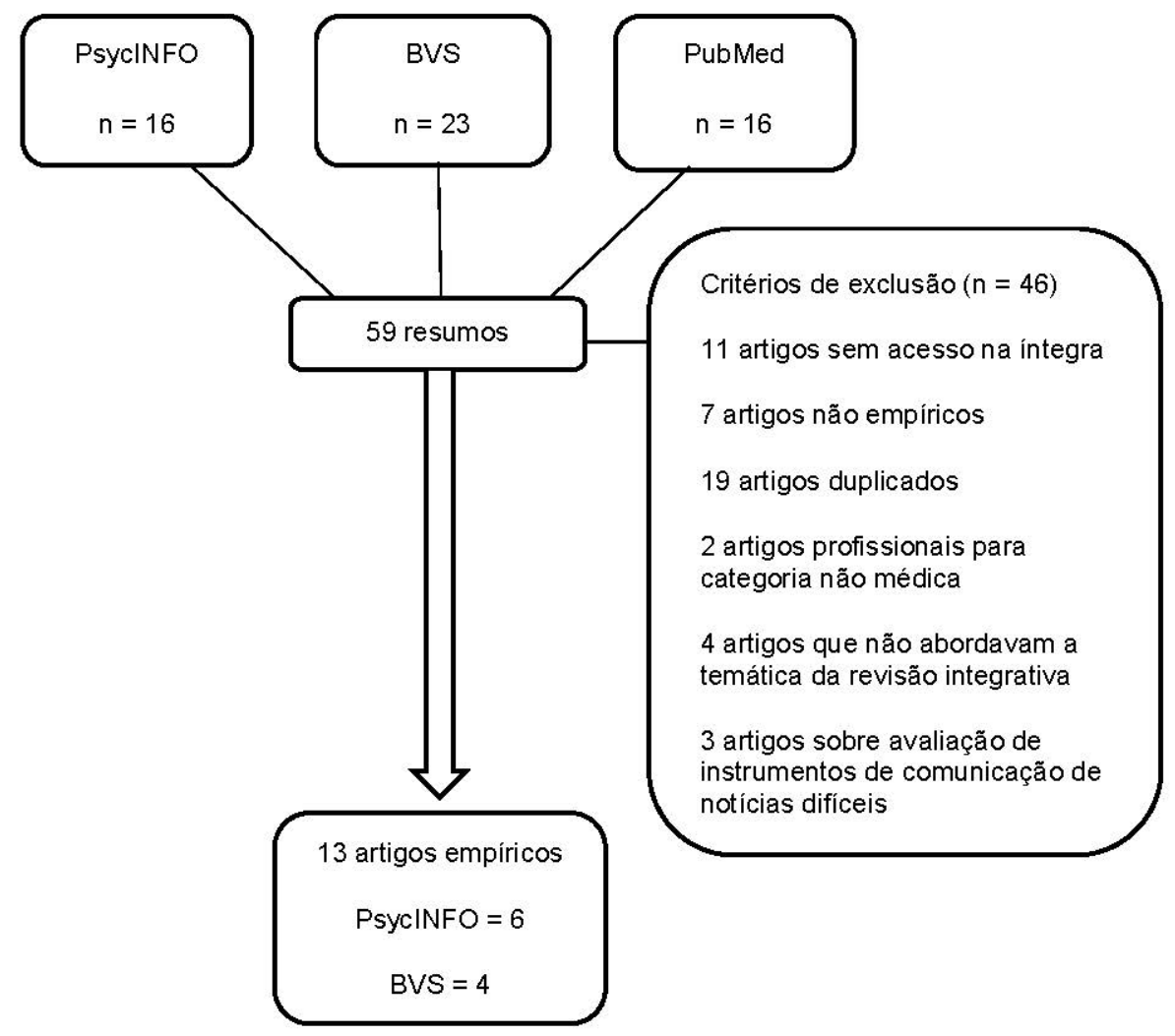

Quanto ao delineamento metodológico, identificaram-se: nove estudos de intervenção com métodos mistos; dois estudos descritivos qualitativos; um estudo longitudinal de coorte com métodos mistos e um estudo transversal quantitativo. No que tange ao perfil dos participantes dos estudos, dez artigos foram realizados com estudantes de medicina e três com médicos residentes. Da amostra analisada, quatro abordaram temáticas de treinamento, capacitação ou formação curricular relacionada à comunicação de notícias difíceis para estudantes e residentes (Johnson et al., 2017; Luttenberger, Graessel, Simon, \& Donath, 2014; Parikh, White, Buckingham, \& Tchorz, 2017; Williams-Reade, Lobo, Whittemore, Parra, \& Baerg, 2018).

Quatro artigos apresentaram teorias como abordagem pedagógica de ensino de comunicação de notícias difíceis, sendo estas: a terapia centrada no cliente de Rogers (Luttenberger et al., 2014), a teoria da aprendizagem adulta de Knowles (Johnson et al., 2017), os quatro estágios experienciais de Kolb associados com a teoria familiar sistêmica (Williams-Reade et al., 2018) e a teoria da metacognição (Lajoie et al., 2015). Todos os artigos sinalizaram a 
importância dos aspectos emocionais na comunicação de notícias difíceis, contudo apenas seis estudos pontuaram a necessidade de acolhimento emocional das angústias, da insegurança e do distanciamento emocional reportados pelos participantes, como forma de prepará-los para lidar com a temática de notícias difíceis (Hurst, Baroffio, Ummel, \& Burn, 2015; Karnieli-Miller, Palombo, \& Meitar, 2018; Lajoie et al., 2015; Skye, Wagenschutz, Steiger, \& Kumagai, 2014; Toivonena et al., 2017; Williams-Reade et al., 2018).

Do total de artigos, 11 pontuaram que os estudos realizados ou descritos decorreram de atividades instituídas nos currículos de formação médica, quer seja na graduação, quer seja nos programas de residência (Hurst et al., 2015; Johnson et al., 2017; Karnieli-Miller et al., 2018; Kron et al., 2016; Luttenberger et al., 2014; Sombra Neto et al., 2017; Parikh et al., 2017; Schmitz et al., 2018; Skye et al., 2014; Toivonena, et al., 2017; Williams-Reade et al., 2018). Esses artigos sinalizaram que, apesar de as exigências de participação nos cursos ou disciplinas constituírem requisitos obrigatórios na formação, a participação nas pesquisas tinha caráter voluntário e aqueles que se abstivessem de participar delas não sofreriam sanções no processo de avaliação das referidas práticas de ensino.

Entre as técnicas de ensino identificadas, os artigos apresentaram uma perspectiva multimodal, trabalhando com duas ou mais estratégias. A simulação (role-plalying) foi a mais citada, sendo descrita em 11 estudos (Baile \& Blatner, 2014; Hurst et al., 2015; Johnson et al., 2017; Karnieli-Miller et al., 2018; Kron et al., 2016; Lajoie et al., 2014; Luttenberger et al., 2014; Sombra Neto et al., 2017; Parikh et al., 2017; Skye et al., 2014; Williams-Reade et al., 2018). Nessa técnica, o papel do paciente ou familiar era desempenhado por atores ou pessoas treinadas, conforme um roteiro padronizado. Desses 11 artigos, em um houve inversão de papéis, de modo que os participantes experienciaram o papel de paciente (Luttenberger et al., 2014).

Quatro estudos empregaram ambiente instrucional multimídia com tecnologias digitais, tais como módulos de ensino on-line, realidade virtual, aprendizagem mediada por computador, discussão em grupo em chats de conversa (Lajoie et al., 2014; Lajoie et al., 2015; Kron et al., 2016, Schmitz et al., 2018). Desses quatro, dois estudos tinham características transculturais e foram realizados em países diversos, como Estados Unidos, Canadá e China (Lajoie et al., 2014; Lajoie et al., 2015). A apresentação de vídeos de casos também foi descrita em três artigos (Lajoie et al., 2014; Lajoie et al., 2015; Schmitz et al., 2018).

Por fim, quatro artigos detalharam a aprendizagem baseada em problemas (ABP) como metodologia norteadora dos trabalhos (Hurst et al., 2015; Johnson et al., 2017; Lajoie et al., 2014; Lajoie et al., 2015). Os demais nove artigos, apesar de não descreverem a metodologia de ABP, apresentaram técnicas ou procedimentos de ensino (simulação com pessoas ou em computador, discussão entre pares, escrita reflexiva, reunião compartilhada com pacientes, vídeos de casos, realidade virtual), focando a apresentação de situações rotineiras ou padronizadas nas áreas estudadas (Tabela 1 ). 
Tabela 1

Dados dos Artigos Analisados na Revisão Integrativa

\begin{tabular}{|c|c|c|c|}
\hline Autores e ano & Objetivo & Delineamento & Estratégia(s) de ensino \\
\hline $\begin{array}{l}\text { Hurst et al. } \\
\text { (2015) }\end{array}$ & $\begin{array}{l}\text { Investigar a influência de uma } \\
\text { intervenção de ensino simulada } \\
\text { com pacientes, integrando a } \\
\text { aprendizagem de habilidades de } \\
\text { comunicação, ética às atitudes } \\
\text { e preocupação em relação à } \\
\text { verdade. }\end{array}$ & $\begin{array}{l}\text { Estudo longitudi- } \\
\text { nal de coorte com } \\
\text { métodos mistos. }\end{array}$ & $\begin{array}{l}\text { Simulação com pacientes } \\
\text { padronizados. }\end{array}$ \\
\hline $\begin{array}{l}\text { Johnson et al. } \\
\text { (2017) }\end{array}$ & $\begin{array}{l}\text { Desenvolver curso de comuni- } \\
\text { cação pediátrica em cuidados } \\
\text { intensivos para residentes em } \\
\text { formação na área. }\end{array}$ & $\begin{array}{l}\text { Estudo de inter- } \\
\text { venção com méto- } \\
\text { dos mistos. }\end{array}$ & $\begin{array}{l}\text { Discussões em grupo, } \\
\text { simulação com atores } \\
\text { especificamente trei- } \\
\text { nados como pais de } \\
\text { pacientes, exercícios } \\
\text { autorreflexivos. }\end{array}$ \\
\hline $\begin{array}{l}\text { Karnieli-Miller et } \\
\text { al. (2018) }\end{array}$ & $\begin{array}{l}\text { Melhorar a compreensão da } \\
\text { aprendizagem na escrita reflexiva } \\
\text { de comunicação de más notícias, } \\
\text { identificando componentes que } \\
\text { inibem esse aprendizado. }\end{array}$ & $\begin{array}{l}\text { Estudo descritivo } \\
\text { qualitativo. }\end{array}$ & $\begin{array}{l}\text { Reunião com pacientes } \\
\text { para compartilhamento } \\
\text { de vivências, escritas } \\
\text { narrativas. }\end{array}$ \\
\hline Kron et al. (2016) & $\begin{array}{l}\text { Comparar a comunicação entre } \\
\text { estudantes de medicina expostos } \\
\text { à simulação em computador com } \\
\text { seres humanos virtuais, com o } \\
\text { módulo multimídia de aprendiza- } \\
\text { do baseado em computador. }\end{array}$ & $\begin{array}{l}\text { Estudo de inter- } \\
\text { venção com méto- } \\
\text { dos mistos. }\end{array}$ & $\begin{array}{l}\text { Apresentação multimí- } \\
\text { dia sobre comunicação, } \\
\text { simulação com realidade } \\
\text { virtual. }\end{array}$ \\
\hline $\begin{array}{l}\text { Lajoie et al. } \\
\text { (2014) }\end{array}$ & $\begin{array}{l}\text { Examinar a facilitação de grupos } \\
\text { interculturais na aprendizagem } \\
\text { baseada em problemas (ABP) } \\
\text { usando ferramentas e vídeos } \\
\text { digitais on-line. }\end{array}$ & $\begin{array}{l}\text { Estudo de inter- } \\
\text { venção com méto- } \\
\text { dos mistos. }\end{array}$ & $\begin{array}{l}\text { Ambiente instrucional } \\
\text { por computador, vídeos } \\
\text { digitais on-line, simula- } \\
\text { ção virtual com paciente. }\end{array}$ \\
\hline $\begin{array}{l}\text { Lajoie et al. } \\
\text { (2015) }\end{array}$ & $\begin{array}{l}\text { Examinar o papel dos processos } \\
\text { regulatórios em estudantes de } \\
\text { medicina que transmitem más } \\
\text { notícias a pacientes, em contex- } \\
\text { to de aprendizagem baseada em } \\
\text { problemas (ABP) na web. }\end{array}$ & $\begin{array}{l}\text { Estudo de inter- } \\
\text { venção com méto- } \\
\text { dos mistos. }\end{array}$ & $\begin{array}{l}\text { Ambiente computacional } \\
\text { para comunicação entre } \\
\text { estudantes e facilitado- } \\
\text { res médicos a distância, } \\
\text { discussão de caso em } \\
\text { ambiente computacional } \\
\text { entre pares, vídeo de } \\
\text { caso. }\end{array}$ \\
\hline $\begin{array}{l}\text { Luttenberger et } \\
\text { al. (2014) }\end{array}$ & $\begin{array}{l}\text { Desenvolver currículo para es- } \\
\text { tudantes de medicina, incluindo } \\
\text { dramatizações sobre anamnese, } \\
\text { tomada de decisão comparti- } \\
\text { lhada, prevenção, comunicação } \\
\text { de más notícias e as chamadas } \\
\text { "interações difíceis". }\end{array}$ & $\begin{array}{l}\text { Estudo de inter- } \\
\text { venção com méto- } \\
\text { dos mistos. }\end{array}$ & $\begin{array}{l}\text { Dramatizações desempe- } \\
\text { nhadas por estudantes } \\
\text { nos papéis de médico e } \\
\text { paciente. }\end{array}$ \\
\hline $\begin{array}{l}\text { Sombra Neto et } \\
\text { al. (2017) }\end{array}$ & $\begin{array}{l}\text { Aprimorar a habilidade de co- } \\
\text { municação de más notícias na } \\
\text { prática médica. }\end{array}$ & $\begin{array}{l}\text { Estudo transversal } \\
\text { quantitativo. }\end{array}$ & $\begin{array}{l}\text { Simulações com pa- } \\
\text { cientes atores, tre- } \\
\text { inamento em pares, } \\
\text { exame clínico objetivo } \\
\text { estruturado. }\end{array}$ \\
\hline
\end{tabular}




\begin{tabular}{llll}
\hline Autores e ano & Objetivo & Delineamento Estratégia(s) de ensino \\
\hline
\end{tabular}

Examinar o acompanhamento

em longo prazo no treinamento (2017) no fim da vida, avaliação e retenção de habilidades em estudan-

Estudo de inter- Simulação com pacientes venção com méto- padronizados. dos mistos. tes de medicina.

Examinar os efeitos da

Schmitz et al. (2018) aprendizagem na comunicação diante de diferentes formatos de abordagem de paciente simulado.

Explorar contextos, experiências e reações em dar e receber más

Skye et al. (2014) notícias no ensino médico, a partir do uso do teatro interativo e da dramatização.

Conhecer as reflexões de esToivonena et al. tudantes de medicina sobre as (2017) emoções no contexto de comunicação de más notícias.

Conduzir treinamento de residentes de cirurgia pediátrica

Williams-Reade et al. (2018) sobre como comunicar compassivamente más notícias de morte pediátrica a familiares.
Abordagem do paciente com texto, abordagem do paciente com versão Estudo de inter- de vídeo e abordagem venção com méto- de paciente com versão dos mistos. de vídeo enriquecida com texto do protocolo Spikes, simulação com paciente.

Estudo de inter- Dramatizações de casos, venção com méto- simulação com pacientes dos mistos. padronizados, discussão em grupo.

Estudo descritivo qualitativo.

Simulação com paciente padronizado e discussão em grupo.

Estudo de intervenção com méto- Simulação com atores, dos mistos.

\section{Discussão}

Vários países discutem a necessidade de se adotar um currículo e uma formação médica adequados à realidade dos sistemas de saúde. Para isso, faz-se necessário articular práticas de assistência ao paciente, conhecimento médico, habilidades interpessoais e de comunicação, profissionalismo e aprendizagem baseada em práticas problematizadoras. Como exemplo disso, citam-se as diretrizes do Conselho de Credenciamento para Educação Médica de Pós-Graduação nos Estados Unidos (Accreditation Council for Graduate Medical Education [ACGME], 2019) e o CanMEDS (2019), estrutura educacional canadense que detalha e apresenta as habilidades médicas necessárias para atendimento efetivo às necessidades de saúde da população. No Brasil, as Diretrizes Curriculares Nacionais (DCNs) dos cursos de medicina também acompanham as mudanças globais nos referenciais educacionais e nas políticas de saúde, primando por uma formação profissional aliada às mudanças contemporâneas da sociedade (Brasil, 2014; Gomes, Brino, Aquilante, \& Silva de Avó, 2009).

Os resultados do presente estudo revelaram uma prevalência de trabalhos realizados com estudantes de graduação, o que vai ao encontro das diretrizes curriculares mencionadas acima. Considerando que essas diretrizes estabelecem os princípios, os fundamentos e as 
finalidades da habilitação médica, elas têm papel norteador no projeto pedagógico de formação. No entanto, foi observada uma escassez de trabalhos com os docentes, peças primordiais no processo de ensino. Segundo Costa e Azevedo (2010), o papel do docente seria o de oferecer experiências que aumentem o autoconhecimento, o poder de escuta, o respeito e a tolerância, auxiliando os estudantes a detectar suas fragilidades, medos e preconceitos. Isso também proporciona ao aluno uma observação mais ampliada da atuação médica, que pode ser terapêutica em si. Daí, depreende-se a importância do papel do professor, não só como transmissor de conhecimento, mas também como multiplicador de modelos assistenciais na prática médica, de modo que esses profissionais necessitam também de aprimoramento e educação permanente em suas atividades docentes no que se refere à comunicação de notícias difíceis.

Apesar de apenas um terço dos artigos detalharem o uso da ABP, o presente estudo identificou que os demais artigos descreveram práticas problematizadoras coerentes com essa metodologia. A ABP trabalha com situações do dia a dia, promovendo a integração entre a teoria e a prática, bem como o desenvolvimento e o aprimoramento de habilidades técnicas, cognitivas, afetivas e de atitudes na formação (Baile \& Blatner, 2014). Para otimizar seu trabalho, ela concilia o emprego de múltiplas técnicas ou estratégias de ensino, tais como as identificadas neste estudo para troca ou compartilhamento da informação. No caso desta RI, mesmo não havendo uma diferenciação clara entre a metodologia ativa e as técnicas empregadas, depreende-se que as estratégias de ensino em saúde, por serem múltiplas, remetem-nos à própria diversidade do campo, pois existem inúmeras formas de se compartilhar as experiências e o conhecimento na área. Além disso, para entrar em contato com a experiência humana na saúde e na doença, essa diversidade também se faz necessária na educação médica.

Os resultados sinalizaram o emprego de tecnologias digitais e de recursos da rede mundial de computadores como forma de mediação do ensino e da aprendizagem. Um ponto identificado no estudo refere-se ao uso dessas tecnologias digitais em situações com distâncias geográficas ou continentais. Isso inclusive reflete o panorama mundial, pois a sociedade contemporânea utiliza-se dessas tecnologias para facilitar as relações de informação e comunicação e, atualmente, não há praticamente nada que não seja intermediado por elas. Isso corrobora a ideia de que a Educação precisa se familiarizar com os recursos tecnológicos para incentivar as atividades pedagógicas, propiciando melhor interação entre os agentes envolvidos no processo de ensino-aprendizagem (Andrade, 2015).

Dos artigos analisados, $46 \%$ sinalizaram a necessidade de acolhimento ao medo, às angústias, à insegurança e ao distanciamento emocional reportados pelos participantes no processo de comunicação de notícias difíceis. Esse dado vai ao encontro da literatura, ao sinalizar que o processo de ensino interatua com afetos e sentimentos, os quais podem promover ou impactar os processos cognitivos envolvidos na comunicação e aprendizagem de modelos de assistência em saúde (Karnieli-Miller et al., 2018; Toivonena et al., 2017; Lajoie, 2015; Hurst et al., 2015). Ceccim (2008) pontua que o campo das construções cognitivas é também espaço de requalificação de afetos (processo de singularização) e de convite às aprendizagens de natureza complexa (processos intelectivos). Contudo, como, usualmente, os métodos educacionais legitimam o saber acumulado e negligenciam as aquisições intensivas (a criação do trabalho coletivo, a interdependência com os usuários, a adoção de 
práticas externas às instituições de ensino e a transdisciplinaridade), a implicação afetiva no espaço de produção do saber acaba sendo denegada na formação.

Diante do exposto, cabe destacar que a formação médica é um importante componente do HumanizaSUS, programa da Política Nacional de Humanização (PNH) do Sistema Único de Saúde (SUS), instituído em 2003 e que visa à execução dos princípios do SUS no cotidiano das práticas de atenção e gestão, qualificando a saúde pública no Brasil. O HumanizaSUS aplica-se a diferentes ações, políticas públicas e instâncias gestoras, demandando trocas comunicativas entre os sujeitos e os cenários envolvidos, tendo por finalidade a valorização e a implicação dos diferentes atores envolvidos no processo de produção de saúde, quer sejam eles usuários, quer sejam eles trabalhadores ou gestores (Brasil, 2020).

\section{Conclusão}

Quanto à formação médica para comunicação de notícias difíceis, esta revisão integrativa identificou que a aprendizagem baseada em problemas (ABP) foi a metodologia ativa mais descrita, enquanto a simulação foi a estratégia de ensino mais empregada. O uso combinado dessas situa o aluno no centro do processo de aprendizagem e o envolve na atividade de ensino, ao favorecer que ele contate situações-problema. Foram identificadas também outras estratégias de ensino (discussão entre pares, escrita reflexiva, reunião compartilhada com pacientes, vídeos de casos e emprego de tecnologias digitais), as quais podem ser aplicadas nos mais diversos cenários, desde que contextualizadas aos objetivos do processo de ensino e aos recursos disponíveis para sua implementação. Reforça-se a importância do uso dessas estratégias como medidas complementares à profissionalização médica contemporânea, contribuindo com vivências ampliadas no processo de ensino-aprendizagem.

Reconhece-se como limitações deste trabalho a falta de artigos com profissionais já habilitados para comunicação de notícias difíceis, assim como com os docentes no ensino desse tipo de comunicação. Isso se faz necessário, pois apresentaria dados pertinentes à educação continuada do profissional e da educação permanente do professor. Além disso, sugere-se para investigação futura a realização de estudos com cada estratégia específica de ensino, de modo a identificar os aspectos fortes, as fragilidades e as possibilidades de aplicação de cada estratégia.

Por fim, sinaliza-se a necessidade de se explorar estudos de comunicação de notícias difíceis na perspectiva da interdisciplinaridade, e não única e meramente na perspectiva médica. Isso porque o levantamento de condutas acerca desse tipo de comunicação por equipes multiprofissionais pode contribuir para a melhoria do compartilhamento de informação entre os atores envolvidos, quer sejam eles pacientes, quer sejam eles familiares ou profissionais, implicando-os nos processos de cuidado. E, quanto a isso, a Psicologia tem muito a contribuir no campo das construções intersubjetivas, da comunicação terapêutica e da humanização na aplicação de modelos de intervenção e de assistência biopsicossociais.

\section{Referências}

Accreditation Council for Graduate Medical Education. (2019). What we do. ACGME Disponível em https://www.acgme.org/What-We-Do/Overview 
Afonso, S. B. C., \& Minayo, M. C. S. (2017). Relações entre oncohematopediatras, mães e crianças na comunicação de notícias difíceis. Ciência \& Saúde Coletiva, 22(1), 53-62. doi: https://doi.org/10.1590/1413-81232017221.14592016

Andrade, E. F. (2015). Tecnologias digitais e ensino. Artefactum - Revista de Estudos em Linguagem e Tecnologia, 7 (1). Disponível em http://artefactum.rafrom.com.br/index. php/artefactum/article/view/667

Araújo, J. A., \& Leitão, E. M. P. (2012). A comunicação de más notícias: Mentira piedosa ou sinceridade cuidadosa. Revista do Hospital Universitário Pedro Ernesto, 11, 58-62. Disponível em http://revista.hupe.uerj.br/detalhe_artigo.asp?id=327

Baile, W. F., Buckman, R., Lenzi, R., Glober, G., Beale, E. A., \& Kudelka, A. P. (2000). SPIKES - Um protocolo em seis etapas para transmitir más notícias: Aplicação ao paciente com câncer. The Oncologist, 5, 302-311. Disponível em https://edisciplinas.usp.br/ pluginfile.php/4686780/mod_resource/content/1/SPIKES\%20\%E2\%80\%93\%20Um\%20 Protocolo\%20em\%20Seis\%20Etapas\%20para\%20Transmitir\%20M\%C3\%A1s\%20 Not\%C3\%ADcias\%20Aplica\%C3\%A7\%C3\%A30\%20ao\%20Paciente\%20co.pdf

Baile, W. F., \& Blatner, A. (2014). Teaching communication skills: Using action methods to enhance role-plays in problem-based learning. Healthcare, 9, 220-227. doi:https://doi. org/10.1097/SIH.0000000000000019

Botelho, L. L. R., Cunha, C. C. de A., \& Macedo, M. (2011). O método da revisão integrativa nos estudos organizacionais. Gestão e Sociedade, 5(11), 121-136. doi:https://doi. org/10.21171/ges.v5i11.1220

Brasil. Conselho Federal de Medicina. (2009). Resolução n. 1931/09. Sobre o Código de Ética Médica. Brasília-DF: CFM. Disponível em http://portal.cfm.org.br/index. php?option=com_content\&id=20670:resolucao-

Brasil. Ministério da Educação. (2014). Resolução n. 03/2014. Institui as diretrizes curriculares nacionais do curso de graduação em Medicina e dá outras providências. Brasília-DF: MEC. Disponível em http://portal.mec.gov.br/index.php? option=com_ docman\&view=download\&alias=15874-rces003-14\&category_slug=junho-2014pdf\&Itemid $=30192$

Brasil. Ministério da Saúde. (2020). HumanizaSUS - Política Nacional de Humanização. BrasíliaDF: MS. Disponível em https://www.saude.gov.br/acoes-e-programas/humanizasus/ sobre-o-programa.

Royal College of Physicians and Surgeons of Canada. (2019). CanMEDS. Disponível em http:// www.royalcollege.ca/rcsite/canmeds/about-canmeds-e

Ceccim, R. B. (2008). A emergência da educação e ensino da saúde: Interseções e intersetorialidade. Revista Ciência e Saúde, 1(1), 9-23. doi:https://doi. org/10.15448/1983-652X.2008.1.3859

Chehuen Neto, J. A., Sirimarco, M. T., Cândido, T. C., Bicalho, T. C., Matos, B. O., Berbert, G. H., \& Vital, L. V. (2013). Profissionais de saúde e a comunicação de más notícias sob a ótica do paciente. Revista Médica de Minas Gerais, 23(4), 518-25. doi:https://doi. org/10.5935/2238-3182.20130079

Costa, F. D., \& Azevedo, R. C. S. (2010). Empatia, relação médico-paciente e formação em medicina: Um olhar qualitativo. Revista Brasileira de Educação Médica, 34(2), 261-269. doi:https://doi.org/10.1590/S0100-55022010000200010 
Cunha, A. G. C. (2010). Dicionário etimológico da língua portuguesa (4a ed.). Rio de Janeiro, RJ: Editora Lexikon.

Diesel, A., Baldez, A. L. S., \& Martins, S. N. (2017). Os princípios das metodologias ativas de ensino: Uma abordagem teórica. Revista Thema, 14(1), 268-288. doi:https://doi. org/10.15536/thema.14.2017.268-288.404

Ercole, F. F., Melo, L. S., \& Alcoforado, C. L. G. C. (2014). Integrative review versus systematic review. Revista Mineira de Enfermagem, 18(1), 102-106. doi:https://doi. org/10.5935/1415-2762.20140001

Geovanini, F., \& Braz, M. (2013). Conflitos éticos na comunicação de más notícias. Revista Bioética, 21(3), 455-462. doi:https://doi.org/10.1590/S1983-80422013000300010

Gomes, R., Brino, R. F., Aquilante, A. G., \& Silva de Avó, L. R. (2009). Aprendizagem Baseada em Problemas na formação médica e o currículo tradicional de Medicina: Uma revisão bibliográfica. Revista Brasileira de Educação Médica, 33(3), 444-451. doi:https://doi. org/10.1590/S0100-55022009000300014

Hurst, S. A., Baroffio, A., Ummel, M., \& Burn, C. L. (2015). Helping medical students to acquire a deeper understanding of truth-telling. Medical Education Online, 20(1). doi:https://doi. org/10.3402/meo.v20.28133

Johnson, E. M., Hamilton, M. F., Watson, R. S., Claxton, R., Barnett, M., Thompson, A. E., \& Arnold, R. (2017). An intensive, simulation-based communication course for pediatric critical care medicine fellows. Online Clinical Investigations, 18(8), 348-355. doi:https:// doi.org/10.1097/PCC.0000000000001241

Karnieli-Miller, O., Palombo, M., \& Meitar, D. (2018). See, reflect, learn more: Qualitative analysis of breaking bad news reflective narratives. Medical Education, 52(5), 497-512. doi:https://doi.org/10.1111/MEDU.13582

Kron, F. W. K., Fetters, M. D., Scerbo, M. W., White, C. B. Lypson, M. L., Padilla, M. A., . . Becker, D. M. (2016). Using a computer simulation for teaching communication skills: A blinded multisite mixed methods randomized controlled trial. Patient Education and Counseling, 100(4), 748-759. doi:https://doi.org/10.1016/j.pec.2016.10.024

Lajoie, S. P., Hmelo-Silver, C., Wiseman, J., Chan, L. K., Lu, J., Khurana, C., . . \& Kazemitabar, M. (2014). Using online digital tools and video to support international problem-based learning. The Interdisciplinary Journal of Problem-based Learning, 8(2), 60-75. doi:https:// doi.org/10.7771/1541-5015.1412

Lajoie, S. P., Lee, L., Poitras, E., Bassiri, M., Kazemitabar, M., Cruz-Panesso, I., . . ., Lu, J. (2015). The role of regulation in medical student learning in small groups: Regulating oneself and others' learning and emotions. Computers in Human Behavior, 52, 601-616. doi:https:// doi.org/10.1016/j.chb.2014.11.073

Lamba, S., Tyrie, L. S., Bryczkowski, S., \& Nagurka, R. (2016). Teaching surgery residents the skills to communicate difficult news to patient and family members: A literature review. Journal of Palliative Medicine, 19 (1), 101-107. doi:https://doi.org/10.1089/ jpm.2015.0292

Luttenberger, K., Graessel, E., Simon, C., \& Donath, C. (2014). From board to bedside Training the communication competences of medical students with role plays. BMC Medical Education, 14, 135-145. doi:https://doi.org/10.1186/1472-6920-14-135 
Paiva, M. R. F., Parente, J. R. F., Brandão, I. R., \& Queiroz, H. B. (2016). Metodologias ativas de ensino-aprendizagem: Revisão integrativa. Sanare, 15(2), 145-153. Disponível em https:// sanare.emnuvens.com.br/sanare/article/view/1049

Parikh, P. P., White, M. T., Buckingham, L., \& Tchorz, K. M. (2017). Evaluation of palliative care training and skills retention by medical students. Journal of Surgical Research, 211, 172177. doi:https://doi.org/10.1016/j.jss.2016.11.006

Robbins, S. P. (2004). Fundamentos do comportamento organizacional (7a ed.). São Paulo, SP: Pearson.

Ruiz R. (2003). Programas de formación en comunicación clínica: Una revisión de su eficacia en el contexto de la enseñanza médica. Educación Médica, 6(4), 159-167. Disponível em http://scielo.isciii.es/pdf/edu/v6n4/original7.pdf

Schmitz, F. M., Schnabel, K. P., Bauer, D., Bachmann, C., Woermann, U., \& Guttormsen, S. (2018). The learning effects of different presentations of worked examples on medical students' breaking-bad-news skills: A randomized and blinded field trial. Patient Education and Counseling, 101(8), 1439-1451. doi:https://doi.org/10.1016/j.pec.2018.02.013

Silva, A. E., Sousa, P. A., \& Ribeiro, R. F. (2018). Comunicação de notícias difíceis: Percepção de médicos que atuam em oncologia. Revista de Enfermagem do Centro-Oeste Mineiro, 8(2482). doi:https://doi.org/10.19175/recom.v7i0.2482

Skye, E. P., Wagenschutz, H., Steiger, J. A., \& Kumagai, A. K. (2014). Use of interactive theater and role play to develop medical students' skills in breaking bad news. Journal of Cancer Education, 29(4), 704-708. doi:https://doi.org/10.1007/s13187-014-0641-y

Sombra Neto, L. L., Silva, V. L. L., Lima, C. D. D., Moura, H. T. M., Gonçalves, A. L. M., Pires, A. P. B., \& Fernandes, V. G. (2017). Habilidade de comunicação da má notícia: O estudante de medicina está preparado? Revista Brasileira de Educação Médica, 41(2), 260-268. doi:https://doi.org/10.1590/1981-52712015v41n2rb20160063

Souza, C. S., Iglesias, A. G., \& Pazin-Filho, A. (2014). Estratégias inovadoras para métodos de ensino tradicionais - Aspectos gerais. Medicina, 47(3), 284-292. doi:https://doi. org/10.11606/issn.2176-7262.v47i3p284-292

Souza, M. T., Silva, M. D., \& Carvalho, R. (2010). Revisão integrativa: O queée como fazer. Einstein (São Paulo), 8(1), 102-106. doi:https://doi.org/10.1590/s1679-45082010rw1134

Stellyes, C. E. V. (2017). Comunicación en el currículo médico. Medicina Universidad Pontificia Bolivariana, 36(1), 59-70. doi:https://doi.org/10.18566/medupb.v36n1.a08

Toivonena, A. K., Lindblom-Ylänneb, S., Louhialaa, P., \& Pyöräläc, E. (2017). Medical students' reflections on emotions concerning breaking bad news. Patient, Education and Counseling, 100(10), 1903-1909. doi:https://doi.org/10.1016/j.pec.2017.05.036

Williams-Reade, J.; Lobo, E.; Whittemore, A. A.; Parra, L. \& Baerg, J. (2018). Enhancing residents' compassionate communication to family members: A family systems breaking bad news simulation. Families, Systems, \& Health, 36(4), 523-527. doi:https://doi. org/10.1037/fsh0000331

Recebido em: 11/07/2019

Última revisão: 11/02/2020

Aceite final: 03/03/2020 


\section{Sobre as autoras:}

Esther Almeida da Silva-Xavier: Psicóloga. Mestranda na linha de pesquisa: Psicologia da Saúde e Processos Clínicos na Universidade de Brasília, Programa de Pós-Graduação em Psicologia Clínica e Cultura, Brasília, DF. E-mail: esther.gea.ex@gmail.com, Orcid: http://orcid.org/0000-0002-0168-9087

Larissa Polejack: Doutora em Processos de Desenvolvimento Humano e Saúde. Professora associada do Programa de Pós-Graduação em Psicologia e Cultura da Universidade de Brasília, Brasília, DF. E-mail: larissapolejack@hotmail.com, Orcid: http://orcid.org/0000-0002-0506-1721

Eliane Maria Fleury Seidl: Doutora em Psicologia. Professora associada do Programa de PósGraduação em Psicologia Clínica e Cultura e do Programa de Pós-Graduação em Bioética, na Faculdade de Ciências da Saúde, da Universidade de Brasília, Brasília, DF. E-mail: eliane.seidl@gmail.com, Orcid: http://orcid.org/0000-0002-1942-5100 
